\title{
Pretreatment magnetic resonance imaging of regional lymph nodes with carcinoembryonic antigen in prediction of synchronous distant metastasis in patients with rectal cancer
}

\author{
Huanhuan Liu ${ }^{1, *}$, Yanfen $\mathrm{Cu}^{1}{ }^{1,}$, Wei Shen ${ }^{2}$, Xingwen Fan ${ }^{3}$, Long Cui ${ }^{2}$, Caiyuan \\ Zhang ${ }^{1}$, Gang Ren ${ }^{1}$, Jihong Fu' ${ }^{2}$, Dengbin Wang ${ }^{1}$ \\ ${ }^{1}$ Department of Radiology, Xinhua Hospital Affiliated to Shanghai Jiaotong University School of Medicine, Shanghai 200092, \\ China \\ ${ }^{2}$ Department of Colorectal and Anal Surgery, Xinhua Hospital Affiliated to Shanghai Jiaotong University School of Medicine, \\ Shanghai 200092, China \\ ${ }^{3}$ Department of Radiation Oncology, Fudan University Shanghai Cancer Center, Shanghai 200032, China \\ *These authors have contributed equally to this work \\ Correspondence to: Wei Shen, e-mail: joanshenwei@126.com \\ Dengbin Wang, e-mail: dbwang8@aliyun.com
}

Keywords: MRI, rectal cancer, lymph node, metastasis, carcinoembryonic antigen

Received: November 05, $2015 \quad$ Accepted: February 23, $2016 \quad$ Published: March 8, 2016

\section{ABSTRACT}

Distant metastasis in patients with rectal cancer remains a problem influencing prognosis. Prediction of synchronous distant metastasis is important for the choice of personalized treatment strategies and postoperative follow-up protocol. So far, there are few studies about the predictive value of MRI features combined with clinical characteristics for synchronous distant metastasis in rectal cancer, especially for the lesions developed within 6 months after surgery. We retrospectively reviewed the pretreatment clinical characteristics and magnetic resonance imaging (MRI) features of 271 patients from January 2010 to December 2011 with pathologically confirmed rectal adenocarcinoma and tried to identify independent risk factors for synchronous distant metastasis. Forty-nine patients $(\mathbf{1 8 . 1 \% )}$ were confirmed to have synchronous distant metastasis. Multivariate logistic regression model demonstrated that the elevated carcinoembryonic antigen (CEA), positive MRI-predicted lymph nodes staging $(\mathrm{mrN})$, and MRI-predicted mesorectal fascia (mrMRF) involvement were independent risk factors. The odd ratios were $\mathbf{1 2 . 2}$ for elevated CEA, 5.4 for mrN1 and $\mathbf{7 . 6}$ for mrN2, and 3.8 for mrMRF involvement, respectively. The accuracy and specificity for predicting synchronous distant metastasis by evaluating the positive $\mathrm{mrN}$ combined with elevated CEA were improved to $87.8 \%$ and $94.6 \%$, respectively. The accuracy, sensitivity and specificity of positive $\mathrm{mrN}$ assessment were $86.1 \%, 71.4 \%$ and $91.7 \%$, respectively using the histopathologic results as the reference standard. Altogether, our findings suggest that pretreatment positive $\mathrm{mrN}$ and elevated CEA are independent risk factors for synchronous distant metastasis in rectal cancer and combination of both could help to recognize the patients with high risk for structuring personalized treatment protocol.

\section{INTRODUCTION}

Colorectal cancer is the third most common cancer in the world and its incidence is on the rise [1]. Since the introduction of total mesorectal excision (TME) and application of chemoradiotherapy (CRT), the local recurrence has been dramatically reduced to less than $10 \%[2,3]$. However, distant metastasis rate for rectal cancer remains constant at $20-50 \%$ [2-5]. For patients with untreated colorectal metastatic lesions, the 5-year survival rate was less than 5\% [6]. However, the 5-year survival rate for patients treated with surgical resection of 
colorectal liver or lung metastasis could increase to $40 \%$ and $56.2 \%$, respectively [7-9]. Thus, the preoperative prediction of synchronous distant metastasis, including the presence of distant metastasis at the initial of diagnosis or development of distant metastasis within 6 months after surgery [10], is especially important. For one thing, the high-risk patients could be suggested for further imaging examination, such as contrasted-enhanced MR or PETCT imaging, in detecting more distant metastases. As the contrasted-enhanced MR or PET-CT examination in China has not been in routine use for distant staging. For another, the high-risk patients should be selective for intensified systemic therapy to improve prognosis. Currently, there are quite a few studies about predictive factors for prognosis in rectal cancer. On one hand, lymph node (LN) metastatic status is considered as one of the most important factors influencing prognosis [11]. As demonstrated in the previous studies based on pathologic assessment for LNs, metastatic LNs including $\mathrm{N}$ staging, ratio of metastatic LNs to retrieved LNs (LNR), distribution or extracapsular invasion have shown the prognostic significance in rectal cancer [12-16]. The MRI has been considered as the imaging modality of choice for the preoperative staging of rectal cancer; however, LNs staging is still challenging for MRI $[17,18]$. The diagnostic accuracy of MRI for assessing the LN metastasis varies from $57 \%$ to $85 \%$ [19]. On the other hand, the serum carcinoembryonic antigen (CEA) is known to be a widely available tumor marker for preoperative evaluation and postoperative detection of distant metastasis in patients with rectal cancer $[20,21]$. Nevertheless, the predictive significance of pretreatment CEA level and the cut-off value for the synchronous distant recurrence have yet to be conclusively determined [21].

So far, to the best of our knowledge, there are few studies about the value of combining mrN staging with CEA level for predicting the synchronous distant metastasis. Therefore, the purpose of the present study was to investigate the value of pretreatment mrN staging combined with CEA level for predicting synchronous distant metastasis, in hope of providing non-invasive method preoperatively to recognize patients with high risk of synchronous distant metastasis precisely forpersonalized treatment, such as metastasectomy or intensification of systemic therapy.

\section{RESULTS}

\section{Patients}

Clinical and radiologic characteristics of patients as well as the associations with distant metastasis were shown in Table 1 . The median age of patients was 61 years (range, 30 to 87 years). Of the 271 patients, 68 patients had elevated CEA levels and 203 had normal CEA levels.
Among the patients, 151 patients underwent curative surgery without neoadjuvant treatment, 29 patients underwent chemotherapy or CRT only and the remaining 91 patients underwent surgery after neoadjuvant treatment. Palliative surgeries were performed in 7 patients of the 91 patients.

\section{Synchronous distant metastases}

Among 271 patients, 49 patients (18.1\%) were confirmed to have synchronous distant metastases, 44/49 patients were detected on CT images or at the time of operation and the other 5 patients showed newly developed liver or lung metastasis within 6 months since surgery. The locations were liver $(n=27)$, lung $(n=7)$, both the liver and lung $(n=9)$, both liver and bone $(n=2)$, both liver and distant LNs $(n=2)$ and peritoneum $(n=2)$. Among the 49 patients, 22 patients with distant metastases were confirmed by pathologic analysis and the other 27 patients were clinically diagnosed based on the CT features.

\section{MRI and pathological assessment of regional LNs}

A total of 1922 regional LNs were detected on rectal MR images in the 271 patients. The short axis diameters of $417 \mathrm{LNs}$ were greater than $5 \mathrm{~mm}$, and $58 \mathrm{LNs}$ were less than $5 \mathrm{~mm}$ in diameter but with irregular borders or mixed signal intensity. There were 172, 53 and 46 patients diagnosed as mrN0, mrN1 and mrN2, respectively. There was almost perfect inter-observer agreement with the mrN staging $(\mathrm{k}=0.817)$.

According to the pathological results, 4129 LNs were harvested and 578 LNs were metastatic. Finally, 150 patients were diagnosed as $\mathrm{pN} 0,68$ were $\mathrm{pN} 1$, and 53 were $\mathrm{pN} 2$.

To avoid the effect of neoadjuvant chemotherapy or CRT on the pathological status of LNs, a total of 151 patients who underwent surgery without neoadjuvant treatment were selected for assessing the diagnostic performance of mrN staging. The accuracy, sensitivity, specificity, positive predictive value (PPV), and negative predictive value (NPV) of MRI for metastatic LNs assessment by using pathological results as the reference standard were $86.1 \%, 71.4 \%, 91.7 \%, 76.9 \%$, and $89.3 \%$, respectively.

\section{Risk factors analysis of pretreatment clinico- radiologic variables for synchronous distant metastasis}

The results of univariate analysis for the correlation between the pretreatment clinic-radiologic parameters with distant metastasis were demonstrated in Table 1. Significant differences were observed in CEA level, 
Table 1: Patient characteristics and associations with synchronous distant metastasis

\begin{tabular}{|c|c|c|c|c|c|}
\hline & \multicolumn{2}{|c|}{ Frequency } & \multicolumn{2}{|c|}{ Metastasis } & \multirow[t]{2}{*}{ P value } \\
\hline & $\mathbf{N}$ & $\%$ & $-(\mathrm{N}, \%)$ & $+(\mathrm{N}, \%)$ & \\
\hline \multicolumn{6}{|l|}{ Pretreatment variables } \\
\hline Gender & & & & & 0.314 \\
\hline Male & 171 & 36.9 & $85(38.3)$ & $15(30.6)$ & \\
\hline Female & 100 & 63.1 & $137(61.7)$ & $34(69.4)$ & \\
\hline Age, years & & & & & 0.378 \\
\hline$<65$ & 159 & 58.7 & $133(59.9)$ & $26(53.1)$ & \\
\hline$\geq 65$ & 112 & 41.3 & $89(40.1)$ & $23(46.9)$ & \\
\hline CEA, ng/ml & & & & & $<0.001$ \\
\hline$<10$ & 203 & 74.9 & $188(84.7)$ & $15(30.6)$ & \\
\hline$\geq 10$ & 68 & 25.1 & $34(15.3)$ & $34(69.4)$ & \\
\hline Tumor height, $\mathrm{cm}$ & & & & & 0.262 \\
\hline$>5$ & 220 & 81.2 & $183(82.4)$ & $37(75.5)$ & \\
\hline$\leq 5$ & 51 & 18.8 & $39(17.6)$ & $12(24.5)$ & \\
\hline Tumor diameter, $\mathrm{cm}$ & & & & & 0.238 \\
\hline$\leq 5$ & 107 & 39.5 & $84(37.8)$ & $23(46.9)$ & \\
\hline$>5$ & 164 & 60.5 & $138(62.2)$ & $26(53.1)$ & \\
\hline mrT staging & & & & & 0.001 \\
\hline Low $\mathrm{T}$ staging & 183 & 67.5 & $160(72.1)$ & $23(46.9)$ & \\
\hline High T staging & 88 & 32.5 & $62(27.9)$ & $26(53.1)$ & \\
\hline mrN staging & & & & & $<0.001$ \\
\hline mrNo & 172 & 63.5 & $162(73.0)$ & $10(20.4)$ & \\
\hline mrN1 & 53 & 19.6 & $35(15.8)$ & $18(36.7)$ & \\
\hline $\mathrm{mrN} 2$ & 46 & 17.0 & 2511.3) & $21(42.9)$ & \\
\hline mrMRF & & & & & $<0.001$ \\
\hline Clear & 234 & 86.3 & $201(90.5)$ & $33(67.3)$ & \\
\hline Involvement & 37 & 13.7 & $21(9.5)$ & $16(32.7)$ & \\
\hline
\end{tabular}

CEA: carcinoembryonic antigen

MRF: mesorectal fascia

$\mathrm{P}<0.05$ indicates a statistically significant difference

mrT staging, mrN staging, and circumferential resection margin status (mrMRF) status for distant metastasis. On multivariate logistic analysis, CEA level, mrN staging and mrMRF status of pretreatment variables remained statistically significant (Table 2).

For the patients without CRT treatment, univariate analysis showed that elevated CEA level, positive mrN and mrMRF involvement were significantly correlated with synchronous distant metastasis. The multivariate logistic regression model showed the elevated CEA level
$[P<0.001$, Odd ratio (OR) $25.29,95 \%$ confidence interval (CI 5.42-117.90)], mrN1 staging $(P=0.001$, OR17.45, 95\% CI 3.25-93.79), and mrN2 staging ( $P<0.001$, OR 52.47, 95\% CI 7.36-373.96) remained statistically significant. For the patients with CRT treatment, CEA level, tumor height, and mrN2 staging were significantly correlated with synchronous distant metastasis on univariate analysis. Elevated CEA level $(P<0.001$, OR 6.10, 95\% CI 2.28-16.28) and mrN2 staging ( $P=0.038$, OR 3.40, 


\begin{tabular}{lcc}
\hline & \multicolumn{2}{c}{ Multivariate analysis } \\
\cline { 2 - 3 } & OR $\mathbf{( 9 5 \% \mathbf { C I } )}$ & P value \\
\hline Pretreatment variables & & \\
CEA, ng/ml & 1 & $<0.001$ \\
$\quad<10$ & $12.2(5.3-28.2)$ & \\
$\geq 10$ & & 0.053 \\
mrT staging & 1 & \\
Low T staging & $2.3(1.0-5.4)$ & \\
High T staging & 1 & $<0.001$ \\
mrN staging & & $<0.001$ \\
mrN0 & & \\
mrN1 & $5.4(2.0-14.7)$ & \\
mrN2 & $7.6(2.8-20.7)$ & 0.007 \\
mrMRF & & \\
Clear & $3.8(1.4-9.7)$ & \\
Involvement & &
\end{tabular}

CEA: carcinoembryonic antigen

MRF: mesorectal fascia

OR: odds ratio

CI: confidence interval

$\mathrm{P}<0.05$ indicates a statistically significant difference

95\% CI 1.07-10.76) remained statistically significant on multivariate logistic analysis.

The accuracy, sensitivity, specificity, PPV and NPV of positive mrN, elevated CEA as well as positive $\mathrm{mrN}$ combined with elevated CEA for predicting distant metastasis were shown in Table 3. Compared with positive $\mathrm{mrN}$ or elevated CEA only, the specificity of the positive $\mathrm{mrN}$ combined with elevated CEA was improved to $94.6 \%$.

The predictive performance of MRI-predicted LN metastasis for pathological LN metastasis was also performed. Among the 151 patients undergoing surgery without neoadjuvant treatment, univariate logistic analysis showed both MRI-predicted LN involvement and pathological LN involvement were significant risk factors for distant metastasis (Table 4).

\section{DISCUSSION}

Our study showed that elevated CEA and positive $\mathrm{mrN}$ were independent risk factors for synchronous distant metastasis in rectal cancer. By combining the elevated CEA with positive $\mathrm{mrN}$, the specificity of predicting synchronous distant metastasis could be improved to
94.6\%. Preoperative identification of high-risk patients for distant metastasis is important because those patients could undergo different treatment strategies, such as metastasectomy or intensified systemic therapy [10]. Recent studies indicated intensification of systemic therapy with neoadjuvant combination chemotherapy before standard treatment is feasible in poor-risk potentially operable rectal cancer with acceptable safety and promising long-term outcomes, for it can eradicate micrometastasis by implementation of a full systemic dose $[22,23]$. In term of metastatic LNs assessment, as the presence of microscopic metastases or inflammatory swelling of LNs, the preoperative metastatic LNs assessment remains a challenging problem for radiologists [19]. In our study, a short axis diameter of greater than $5 \mathrm{~mm}$, irregular borders or mixed signal intensity was used as the diagnostic criteria for metastatic LNs. The relatively high diagnostic accuracy of $86.1 \%$ in our study was consistent with that reported by Al-Sukhni et al. [18]. The specificity for metastatic LNs assessment was improved to $91.7 \%$ in our study, indicating that patients without LN involvement could be well identified. Based on the diagnostic criteria, our results demonstrated pretreatment $\mathrm{mrN}$ staging could perform as a risk factor 
Table 3: Predictive performance of pretreatment positive mrN and CEA level for synchronous distant metastasis

\begin{tabular}{|c|c|c|c|c|c|}
\hline Risk factors & Accuracies (\%) & Sensitivities $(\%)$ & Specificities (\%) & PPVs (\%) & NPVs $(\%)$ \\
\hline mrN positive & 74.2 & 79.6 & 73.0 & 39.5 & 94.2 \\
\hline Elevated CEA & 81.9 & 69.4 & 84.7 & 50.0 & 92.6 \\
\hline mrN positive and elevated CEA & 87.8 & 57.1 & 94.6 & 70.0 & 90.9 \\
\hline
\end{tabular}

CEA: carcinoembryonic antigen

PPV: positive predictive value

NPV: negative predictive value

Table 4: Risk of pretreatment positive $\mathrm{mrN}$ and positive $\mathrm{pN}$ for synchronous distant metastasis

\begin{tabular}{llrr}
\hline Findings* & Frequency $(\%) * *$ & Univariate analysis \\
\cline { 3 - 4 } & & OR (95\% CI) & P value \\
\hline mrN positive vs. mrN negative & $11 / 49(22.4)$ vs. 8/112(7.1) & $5.11(1.88-13.91)$ & 0.001 \\
pN positive vs. pN negative & $12 / 42(28.6)$ vs.7/109 (6.4) & $5.83(2.11-16.12)$ & 0.001 \\
\hline
\end{tabular}

mrN: MRI-predicted lymph node

$\mathrm{pN}$ : pathologic lymph node

OR: odds ratio

CI: confidence interval

*A vs. B means that risk of A was analyzed compared to B as the reference standard

$* * a / b$ means the number of patients with findings and metastasis/the number of patients with findings

for synchronous distant metastasis. Sohn et al. [10] also reported that positive regional $\mathrm{LN}$ metastasis on MRI was the highest risk factor for predicting synchronous distant metastasis, compared with the other independent predictors of mrT staging, mrN staging and MRI-detected extramural vascular invasion. However, the performance for the single risk factor of positive $\mathrm{mrN}$ in predicting synchronous distant metastasis in our study was moderate. The accuracy, sensitivity, and specificity were $74.2 \%$, $79.6 \%$, and $73.0 \%$, respectively. To improve the predictive value of pretreatment variables, we tried to predict distant metastasis by combining positive $\mathrm{mrN}$ with another independent predictor of CEA in our study.

The CEA plays a key role in biological phenomena in tumor cells, including adhesion, immunity, and apoptosis [24]. The clinical significance of post-treatment CEA levels in predicting tumor response after preoperative CRT and detecting recurrence after surgery in rectal cancer have been evaluated in the previous studies $[25,26]$. However, the clinical value of pretreatment CEA level and the cut-off value in predicting distant recurrence have come to no conclusion. Mareno Garcia et al. [27] found a pretreatment CEA level $\geq 2.5 \mathrm{ng} / \mathrm{ml}$ was significantly associated with lower disease-free survival and increased recurrences. Wang et al. [21] reported the elevated pretreatment $C E A \geq 5.0 \mathrm{ng} / \mathrm{ml}$ had significantly higher synchronous metastasis. In our study, a cut-off value of $10 \mathrm{ng} / \mathrm{ml}$ was selected and results demonstrated the elevated CEA level was an independent risk factor for predicting synchronous distant metastasis. The elevated pretreatment CEA level had a significantly higher risk for distant metastasis than the normal CEA level. By combining the CEA level with mrN staging, although the sensitivity for predicting distant recurrence was relatively moderate, the specificity was improved to $94.6 \%$. For the high-risk patients for synchronous distant metastasis, the liver contrast-enhanced MRI or PET-CT should be recommended for further detecting metastatic lesions.

In addition, mrMRF was also an independent predictor for distant metastasis in our study. Previous studies reported that mrMRF status was an important prognostic factor for rectal cancer but with different diagnostic criterion [28, 29]. Taylor et al. demonstrated that mrMRF involvement was significantly associated with distant metastatic disease with $1 \mathrm{~mm}$ as the diagnostic criterion [30]. Sohn et al. [10] using the $2 \mathrm{~mm}$ as the diagnostic criterion showed that mrMRF involvement was a risk factor for distant metastasis but not an independent one. In our study, the cut-off value of $1 \mathrm{~mm}$ was used was the diagnostic criterion. However, all the patients with MRI-predicted involvement underwent preoperative neoadjuvant CRT before surgery. Since the application 
of neoadjuvant CRT would affect the pathologic CRM status, the diagnostic performance of MRI-predicted MRF involvement could not be assessed for the lack of reference standard.

Our study has several limitations. Firstly, the diagnostic performance of mrN staging was assessed among the patients without neoadjuvant treatment. Some cases of MRI-predicted LNs involvement with preoperative chemotherapy were excluded, which could lead to selection bias. Furthermore, we tried our best to perform radiological-pathological one-to-one matching of LNs. However, complete matching was difficult to be accomplished. Secondly, MRI-predicted extramural vascular invasion (mrEMVI) is known to be a poor prognostic factor in rectal cancer. However, due to our retrospective study, the imaging resolution was not high enough for vessels analysis. It was difficult for us to differentiate the mrEMVI from the cordlike signal intensity of tumor, the tumor deposit, and a benign desmoplastic reaction. Finally, contrast-enhanced chest and abdominal CT was performed for distant metastasis in our study. The sensitivity of CT imaging in detecting liver metastases was lower than the MRI, which led to the missed lesions in some cases. Therefore, the contrastenhanced MRI should be introduced for detecting the distant metastasis in the future.

In conclusion, positive $\mathrm{mrN}$ and elevated CEA level are independent risk factors for synchronous distant metastasis and combination of both could help to recognize patients with high risk for structuring the personalized treatment protocol.
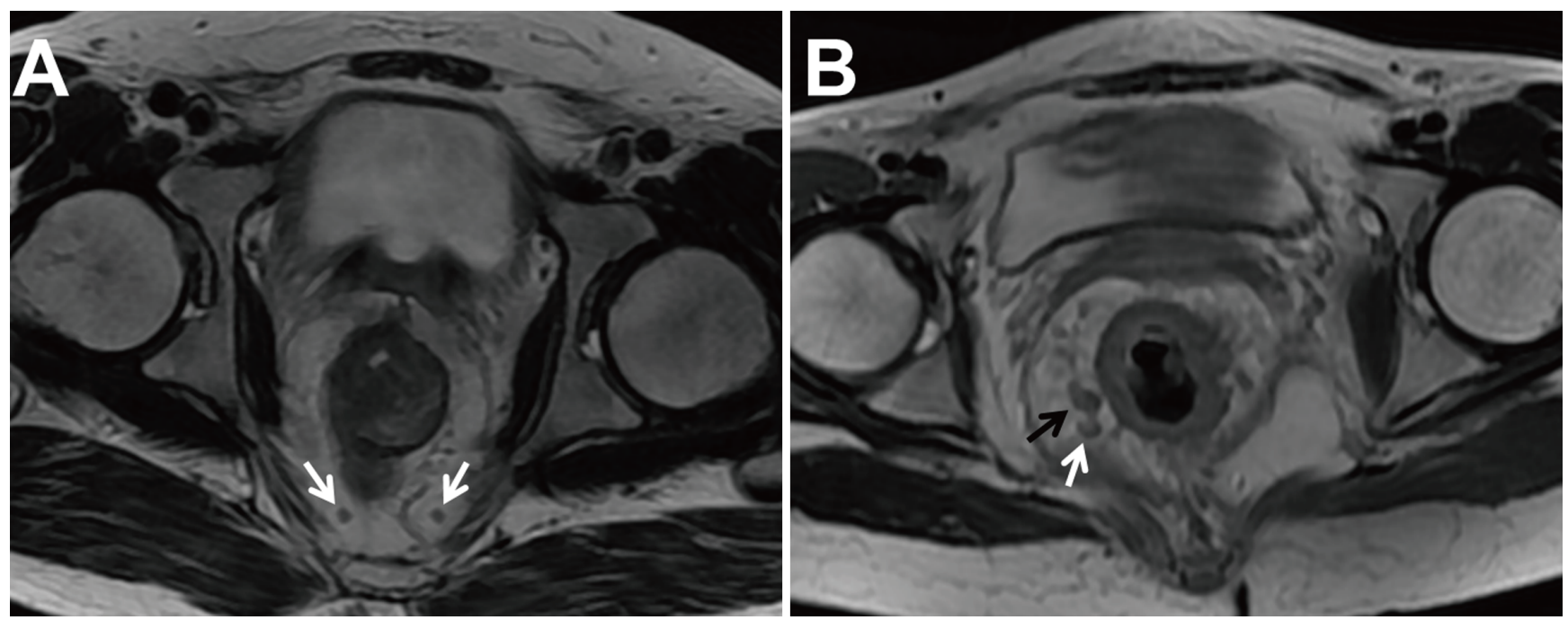

Figure 1: Two T2-weighted axial images in different patients with low rectal cancers. A. Two lymph nodes (LN) within mesorectal fascia with the short axis diameter of 2.5 and $3.0 \mathrm{~mm}$, regular borders, and homogenous signal intensity were considered as non-metastatic LNs (white arrow). B. The LN within mesorectal fascia with the short axis diameter of $6.3 \mathrm{~mm}$ and mixed signal intensity was considered metastatic (black arrow). Another LN with the short axis diameter less than $5 \mathrm{~mm}(4.2 \mathrm{~mm})$ but with mixed signal intensity was also considered metastasis (white arrow).

www.impactjournals.com/oncotarget informed consent from the patients was waived. Data of 331 patients with rectal cancer confirmed by pathological examination from January 2010 to December2011 in our institution and with complete radiologic data were retrospectively reviewed. At the time of initial diagnostic workup, every enrolled patient underwent radiologic examination including pretreatment pelvic MRI and contrast-enhanced chest-abdominal CT. The exclusion criteria were history of polypectomy treatment $(n=39)$, previous pelvic malignancy $(n=16)$ or follow-up loss $(n=5)$. Finally, a total of 271 patients were recruited for analysis.

\section{Imaging protocol}

After bowel preparation, all patients underwent pelvic MRI examinations with 3.0-T system (GE Medical System, Milwaukee, WI, USA) using eight-channel body phased array coil. Oblique axial, sagittal, and coronal T2-weighted MRI were obtained for all the patients. The parameters were as follows: echo time (TE)/repetition time (TR) of 102/4600, 102/4600, and 102/2780, respectively; thickness of $3 \mathrm{~mm}$, slice interval of $1 \mathrm{~mm}$, matrix of 256 $\times 256$, field of view of $25 \times 25$ to $28 \times 28 \mathrm{~cm}$. In our institution, we kept to this protocol for every rectal cancer

MATERIALS AND METHODS

\section{Patients}

This retrospective study was approved by the

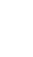


patient for staging and all the patients also underwent contrast-enhanced chest and abdominal CT examinations for detecting distant metastasis on a dual-source multidetector CT (Siemens Somatom Definition Flash, Siemens Medical Solution, Forchheim, Germany).

\section{Image analysis}

A computerized radiologic database was used for image analysis. Two radiologists (with 12 and 15 years of experience in interpreting gastrointestinal tumors, respectively) who were blinded to histopathological results performed the MRI features of rectal cancer in consensus. The disputes between the radiologists were resolved by consultation with a third experienced radiologist with 18 years of experience in interpreting gastrointestinal tumors. Pretreatment MRI features included tumor height from anal verge, the longitudinal diameter on sagittal T2-weighted image, mrT and $\mathrm{mrN}$ staging, and mrMRF. The mrT and mrN staging was defined according to the $7^{\text {th }}$ American Joint Committee on Cancer (AJCC) TNM staging system. T1 and T2 staging was categorized as T1-2 when the tumor was limited within the muscularis propria. T3 staging was defined when the tumor grew through the muscularis propria and into the mesorectum with plaque, mass or cordlike signal intensity projecting into perirectal fat. Tumor penetrating the visceral peritoneum or extending into adjacent organs was considered T4 staging [31]. T1-2 and T3 with extramural spread of at most 5 $\mathrm{mm}$ were considered as low T stage, whereas $\mathrm{T} 3$ with extramural spread greater than $5 \mathrm{~mm}$ and $\mathrm{T} 4$ were regarded as high $\mathrm{T}$ stage. The MRF was considered involvement if the shortest distance of the tumor or metastatic LNs to the MRF was less than $1 \mathrm{~mm} \mathrm{[30].} \mathrm{One} \mathrm{to} \mathrm{three} \mathrm{regional} \mathrm{LNs}$ metastases were defined as mrN1staging, and more than regional LNs metastases were defined as mrN2 staging [32]. Regional metastatic LN involvement was defined as a short axis greater than $5 \mathrm{~mm}$, mixed signal intensity, or irregular borders [10] (Figure 1).

\section{Carcinoembryonic antigen (CEA) and pathological assessment}

Pretreatment serum CEA values were acquired for each patient. CEA level higher than the upper normal limit ( $>10 \mathrm{ng} / \mathrm{mL}$ ) in our institution was considered to be clinically elevated.

Surgeries followed by preoperative CRT were performed 4-8 weeks after neoadjuvant treatment. TME or partial mesorectal excision was completed by anterior resection, abdominoperineal resection or hartmann resection according to the distance of the tumor from the anal verge and sphincteric function. After histological examination, histologic types and grading, depth of tumor invasion, number of retrieved LNs, number of LNs metastasis and circumferential resection margin status (pCRM) were acquired.

\section{Follow-up}

All patients were followed with rigid proctoscopy and serum CEA every 3 months for the first 2 years. Initial multi-detector CT was performed for chest-abdomenpelvis at approximately 6 months since operation.

\section{Statistical analysis}

Statistical analysis was performed with SPSS 19.0 (IBM, New York, NY) and $\mathrm{P}<0.05$ indicated a statistically significant difference. The Cohen $\mathrm{k}$ value was used for evaluation of inter-observer agreement for $\mathrm{mrN}$ staging. The inter-observer agreement was defined as no agreement $(<0.00)$, slight agreement $(0.00-0.20)$, fair agreement (0.21-0.40), moderate agreement (0.41-0.60), substantial agreement (0.61-0.80), and almost perfect agreement (0.81-1.00) [33].

Univariate association of pretreatment clinicoradiologic variables with the status of synchronous distant metastasis was assessed by using the chi-squared test. Multivariate binary logistic regression model was performed to identify independent risk factors for variables with $\mathrm{P}<0.05$ in univariate analysis by using an entry method. Then, the univariate analyses by using the chi-squared test and multivariate logistic regression model by using an entry method were also performed to identify the predictive value of pretreatment clinicoradiologic variables for the synchronous distant metastasis in different grouped patients based on the application of CRT or not. The predictive value of positive $\mathrm{mrN}$ was compared with the pathologic results performed by univariate logistic analysis.

The accuracy, sensitivity, specificity, PPV and NPV of $\mathrm{mrN}$ staging were calculated by using the pathologic results as the reference standard. The accuracy, sensitivity, specificity, PPV and NPV of positive mrN, elevated CEA, as well as positive mrN combined with CEA for predicting synchronous distant metastasis were also calculated.

\section{FUNDING}

This work was supported by the fund of National Nature Science Foundation of China (NSFC No. 81371621, No. 81171389, No. 81372636), and the Program of Shanghai Municipal Health Outstanding Discipline Leader (No. XBR 2013110).

\section{CONFLICTS OF INTEREST}

All authors have no conflicts of interest to disclose. 


\section{REFERENCES}

1. Cho EY, Kim SH, Yoon JH, Lee Y, Lim YJ, Kim SJ, Baek HJ, Eun CK. Apparent diffusion coefficient for discriminating metastatic from non-metastatic lymph nodes in primary rectal cancer. Eur J Radiol. 2013; 82:e662-668.

2. Peeters KC, Marijnen CA, Nagtegaal ID, Kranenbarg EK, Putter H, Wiggers T, Rutten H, Pahlman L, Glimelius B, Leer JW, van de Velde CJ. The TME trial after a median follow-up of 6 years: increased local control but no survival benefit in irradiated patients with resectable rectal carcinoma. Ann Surg. 2007; 246:693-701.

3. Bosset JF, Collette L, Calais G, Mineur L, Maingon P, Radosevic-Jelic L, Daban A, Bardet E, Beny A, Ollier JC. Chemotherapy with preoperative radiotherapy in rectal cancer. N Engl J Med. 2006; 355:1114-1123.

4. Tjandra JJ, Chan MK. Follow-up after curative resection of colorectal cancer: a meta-analysis. Dis Colon Rectum. 2007; 50:1783-1799.

5. Rasanen M, Carpelan-Holmstrom M, Mustonen H, RenkonenSinisalo L, Lepisto A. Pattern of rectal cancer recurrence after curative surgery. Int J Colorectal Dis. 2015; 30:775-785.

6. Simmonds PC. Palliative chemotherapy for advanced colorectal cancer: systematic review and meta-analysis. Colorectal Cancer Collaborative Group. BMJ. 2000; 321:531-535.

7. Kanas GP, Taylor A, Primrose JN, Langeberg WJ, Kelsh MA, Mowat FS, Alexander DD, Choti MA, Poston G. Survival after liver resection in metastatic colorectal cancer: review and meta-analysis of prognostic factors. Clin Epidemiol. 2012; 4:283-301.

8. Kanzaki R, Higashiyama M, Oda K, Fujiwara A, Tokunaga T, Maeda J, Okami J, Tanaka K, Shingai T, Noura S, Ohue M, Kodama K. Outcome of surgical resection for recurrent pulmonary metastasis from colorectal carcinoma. Am J Surg. 2011; 202:419-426.

9. Maguire A, Sheahan K. Controversies in the pathological assessment of colorectal cancer. World J Gastroenterol. 2014; 20:9850-9861.

10. Sohn B, Lim JS, Kim H, Myoung S, Choi J, Kim NK, Kim MJ. MRI-detected extramural vascular invasion is an independent prognostic factor for synchronous metastasis in patients with rectal cancer. Eur Radiol. 2015; 25:1347-1355.

11. Wibe A, Law WL, Fazio V, Delaney CP. Tailored rectal cancer treatment - a time for implementing contemporary prognostic factors? Colorectal Dis. 2013; 15:1333-1342.

12. Vlad C, Kubelac P, Vlad D, Irimie A, Achimas Cadariu P. Evaluation of clinical, morphopathological and therapeutic prognostic factors in rectal cancer. Experience of a tertiary oncology center. J BUON. 2015; 20:92-99.

13. Boras Z, Kondza G, Sisljagic V, Busic Z, Gmajnic R, Istvanic T. Prognostic factors of local recurrence and survival after curative rectal cancer surgery: a single institution experience. Coll Antropol. 2012; 36:1355-1361.
14. Junginger T, Goenner U, Lollert A, Hollemann D, Berres $\mathrm{M}$, Blettner $\mathrm{M}$. The prognostic value of lymph node ratio and updated TNM classification in rectal cancer patients with adequate versus inadequate lymph node dissection. Tech Coloproctol. 2014; 18:805-811.

15. Huh JW, Kim YJ, Kim HR. Distribution of lymph node metastases is an independent predictor of survival for sigmoid colon and rectal cancer. Ann Surg. 2012; 255:70-78.

16. Komori K, Kanemitsu Y, Kimura K, Sano T, Ito S, Abe T, Senda Y, Shimizu Y. Detailed stratification of TNM stage III rectal cancer based on the presence/absence of extracapsular invasion of the metastatic lymph nodes. Dis Colon Rectum. 2013; 56:726-732.

17. Kijima S, Sasaki T, Nagata K, Utano K, Lefor AT, Sugimoto H. Preoperative evaluation of colorectal cancer using CT colonography, MRI, and PET/CT. World J Gastroenterol. 2014; 20:16964-16975.

18. Al-Sukhni E, Milot L, Fruitman M, Beyene J, Victor JC, Schmocker S, Brown G, McLeod R, Kennedy E. Diagnostic accuracy of MRI for assessment of T category, lymph node metastases, and circumferential resection margin involvement in patients with rectal cancer: a systematic review and meta-analysis. Ann Surg Oncol. 2012; 19:2212-2223.

19. Ogawa S, Itabashi M, Hirosawa T, Hashimoto T, Bamba Y, Kameoka S. A logistic model including risk factors for lymph node metastasis can improve the accuracy of magnetic resonance imaging diagnosis of rectal cancer. Asian Pac J Cancer Prev. 2015; 16:707-712.

20. Tan KK, Lopes Gde L, Jr., Sim R. How uncommon are isolated lung metastases in colorectal cancer? A review from database of 754 patients over 4 years. J Gastrointest Surg. 2009; 13:642-648.

21. Wang L, Zhong XG, Peng YF, Li ZW, Gu J. Prognostic value of pretreatment level of carcinoembryonic antigen on tumour downstaging and early occurring metastasis in locally advanced rectal cancer following neoadjuvant radiotherapy (30 Gy in 10 fractions). Colorectal Dis. 2014; 16:33-39.

22. Chua YJ, Barbachano Y, Cunningham D, Oates JR, Brown G, Wotherspoon A, Tait D, Massey A, Tebbutt NC, Chau I. Neoadjuvant capecitabine and oxaliplatin before chemoradiotherapy and total mesorectal excision in MRIdefined poor-risk rectal cancer: a phase 2 trial. Lancet Oncol. 2010; 11:241-248.

23. Glynne-Jones R, Anyamene N, Moran B, Harrison M. Neoadjuvant chemotherapy in MRI-staged high-risk rectal cancer in addition to or as an alternative to preoperative chemoradiation? Ann Oncol. 2012; 23:2517-2526.

24. Hammarstrom S. The carcinoembryonic antigen (CEA) family: structures, suggested functions and expression in normal and malignant tissues. Semin Cancer Biol. 1999; 9:67-81. 
25. Das P, Skibber JM, Rodriguez-Bigas MA, Feig BW, Chang GJ, Wolff RA, Eng C, Krishnan S, Janjan NA, Crane CH. Predictors of tumor response and downstaging in patients who receive preoperative chemoradiation for rectal cancer. Cancer. 2007; 109:1750-1755.

26. Lee JH, Kim DY, Kim SH, Cho HM, Shim BY, Kim TH, Kim SY, Baek JY, Oh JH, Nam TK, Yoon MS, Jeong JU, Kim K, et al. Carcinoembryonic antigen has prognostic value for tumor downstaging and recurrence in rectal cancer after preoperative chemoradiotherapy and curative surgery: A multi-institutional and case-matched control study of KROG 14-12. Radiother Oncol. 2015; 116:202-208.

27. Moreno Garcia V, Cejas P, Blanco Codesido M, Feliu Batlle J, de Castro Carpeno J, Belda-Iniesta C, Barriuso J, Sanchez JJ, Larrauri J, Gonzalez-Baron M, Casado E. Prognostic value of carcinoembryonic antigen level in rectal cancer treated with neoadjuvant chemoradiotherapy. Int $\mathrm{J}$ Colorectal Dis. 2009; 24:741-748.

28. Nagtegaal ID, Quirke P. What is the role for the circumferential margin in the modern treatment of rectal cancer? J Clin Oncol. 2008; 26:303-312.
29. Oberholzer K, Junginger T, Heintz A, Kreft A, Hansen T, Lollert A, Ebert M, Duber C. Rectal Cancer: MR imaging of the mesorectal fascia and effect of chemoradiation on assessment of tumor involvement. J Magn Reson Imaging. 2012; 36:658-663.

30. Taylor FG, Quirke P, Heald RJ, Moran BJ, Blomqvist L, Swift IR, Sebag-Montefiore D, Tekkis P, Brown G. Preoperative magnetic resonance imaging assessment of circumferential resection margin predicts disease-free survival and local recurrence: 5-year follow-up results of the MERCURY study. J Clin Oncol. 2014; 32:34-43.

31. Zhang XM, Zhang HL, Yu D, Dai Y, Bi D, Prince MR, Li C. 3-T MRI of rectal carcinoma: preoperative diagnosis, staging, and planning of sphincter-sparing surgery. AJR Am J Roentgenol. 2008; 190:1271-1278.

32. Kaur H, Choi H, You YN, Rauch GM, Jensen CT, Hou P, Chang GJ, Skibber JM, Ernst RD. MR imaging for preoperative evaluation of primary rectal cancer: practical considerations. Radiographics. 2012; 32:389-409.

33. Lv P, Lin XZ, Chen K, Gao J. Spectral CT in patients with small HCC: investigation of image quality and diagnostic accuracy. Eur Radiol. 2012; 22:2117-2124. 